Pacific

Journal of

Mathematics

\title{
A GEOMETRIC EQUATION WITH CRITICAL NONLINEARITY ON THE BOUNDARY
}

Veronica Felli ANd Mohameden Ould Ahmedou 


\title{
A GEOMETRIC EQUATION WITH CRITICAL NONLINEARITY ON THE BOUNDARY
}

\author{
Veronica Felli And Mohameden Ould Ahmedou
}

\begin{abstract}
A theorem of Escobar asserts that if a three-dimensional smooth compact Riemannian manifold $M$ with boundary is of positive type and is not conformally equivalent to the standard three-dimensional ball, a necessary and sufficient condition for a $C^{2}$ function $H$ on $M$ to be the mean curvature of some conformal scalar flat metric is that $H$ be positive somewhere. We show that, when the boundary is umbilic and the function $H$ is positive everywhere, all such metrics stay in a compact set with respect to the $C^{2}$ norm and the total degree of all solutions is $\mathbf{- 1}$.
\end{abstract}

\section{Introduction}

José F. Escobar [1992a] raised the following question: When is a compact Riemannian manifold with boundary conformally equivalent to one that has zero scalar curvature and whose boundary has constant mean curvature? This problem can be seen as a generalization to higher dimensions of the Riemann Mapping Theorem, which says that an open, simply connected proper subset of the plane is conformally diffeomorphic to the disk. In higher dimensions few regions are conformally diffeomorphic to the ball. However one can still ask whether a domain is conformal to a manifold that resembles the ball in two ways: namely, it has zero scalar curvature and its boundary has constant mean curvature. Escobar's problem is equivalent to seeking a smooth positive solution $u$ to the following nonlinear boundary value problem on an $n$-dimensional Riemannian manifold with boundary $\left(M^{n}, g\right)$, where $n \geq 3$ :

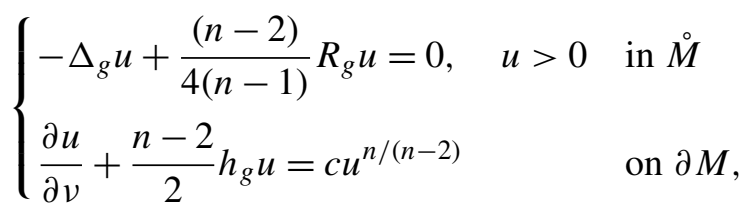

where $R_{g}$ is the scalar curvature of $M, h_{g}$ is the mean curvature of $\partial M, v$ is the outer normal vector with respect to $g$, and $c$ is a constant whose sign is uniquely determined by the conformal structure.

MSC2000: 35J60, 53C21.

Felli is supported by MURST under the national project "Variational Methods and Nonlinear Differential Equations". Ould Ahmedou was supported by a postdoctoral fellowship from SISSA. 
For almost all manifolds, Escobar [1992a; 1996] established that (P) has a solution. More recently in [Ould Ahmedou 2003] this problem was studied using the tool of critical points at infinity developed by A. Bahri [1989] (see also [Bahri and Coron 1988; Bahri and Brezis 1996]). Going beyond the existence results of [Ould Ahmedou 2003], we proved in [Felli and Ould Ahmedou 2003] that, when $(M, g)$ is locally conformally flat with umbilic boundary but not conformal to the standard ball, all solutions of $(\mathrm{P})$ stay in a compact set with respect to the $C^{2}$ norm and the total degree of all solutions is -1 .

The heart of the proof of the result above is some fine analysis of the possible blow-up behaviour of solutions to $(\mathrm{P})$. More specifically, we obtained energyindependent estimates of solutions to

$$
\begin{cases}L_{g} u=0, \quad u>0 & \text { in } \stackrel{\circ}{M}, \\ B_{g} u=(n-2) u^{q} & \text { on } \partial M,\end{cases}
$$

where

$$
1<1+\varepsilon_{0} \leq q \leq \frac{n}{n-2}, \quad L_{g}=\Delta_{g}-\frac{n-2}{4(n-1)} R_{g}, \quad B_{g}=\frac{\partial}{\partial v_{g}}+\frac{n-2}{2} h_{g} .
$$

Instead of looking for conformal metrics with zero scalar curvature and constant mean curvature as in $(\mathrm{P})$, one may also look for scalar-flat conformal metrics with boundary mean curvature being a given function $H$; this problem is equivalent to finding a smooth positive solution $u$ to

$$
\begin{cases}L_{g} u=0, \quad u>0 & \text { in } \stackrel{\circ}{M}, \\ B_{g} u=H u^{n /(n-2)} & \text { on } \partial M .\end{cases}
$$

Such a problem was studied by Escobar [1996], who proved that if a positive three-dimensional smooth compact Riemannian manifold $M$ is not conformally equivalent to the standard three-ball, a necessary and sufficient condition for a $C^{2}$ function $H$ on $M$ to be the mean curvature of some conformal flat metric is that $H$ be positive somewhere. Recall that a manifold is called of positive type, or simply positive, if the quadratic part of the Euler functional associated to $(\mathrm{P})$ is positive definite.

In our work we assume that the boundary is umbilic, that is, the traceless part of the second fundamental form vanishes on the boundary. Moreover we assume that the function $H$ is positive.

Consider for $1<q \leq 3$ the problem

$$
\begin{cases}L_{g} u=0, \quad u>0 & \text { in } \stackrel{\circ}{M}, \\ B_{g} u=H u^{q} & \text { on } \partial M .\end{cases}
$$

We use $M_{H, q}$ to denote the set of solutions of $\left(\mathrm{P}_{H, q}\right)$ in $C^{2}(M)$. Our first theorem gives a priori estimates of solutions of $\left(\mathrm{P}_{H, q}\right)$ in $H^{1}(M)$ norm. 
Theorem 1.1. Let $(M, g)$ be a three-dimensional smooth compact Riemannian manifold with umbilic boundary. Then, for all $\varepsilon_{0}>0$,

$$
\|u\|_{H^{1}(M)} \leq C \quad \text { for all } u \in \bigcup_{1+\varepsilon_{o} \leq q \leq 3} M_{H, q},
$$

where $C$ depends only on $M, g, \varepsilon_{0},\|H\|_{C^{2}(\partial M)}$, and the positive lower bound of $H$.

Our next theorem states that for any positive $C^{2}$ function $H$, all such metrics stay bounded with respect to the $C^{2}$ norm and the total Leray-Schauder degree of all the solutions of $\left(\mathrm{P}_{H, q}\right)$ is -1 .

Theorem 1.2. Let $(M, g)$ be a positive three-dimensional smooth compact Riemannian manifold with umbilic boundary which is not conformally equivalent to the standard three-dimensional ball. Then, for any $1<q \leq 3$ and positive function $H \in C^{2}(\partial M)$, there exists some constant $C$ (depending only on $M, g,\|H\|_{C^{2}}$, the positive lower bound of $H$, and $q$ ) such that

$$
\frac{1}{C} \leq u \leq C \quad \text { and } \quad\|u\|_{C^{2}(M)} \leq C
$$

for all solutions $u$ of $\left(\mathrm{P}_{H, q}\right)$. The total degree of all solutions of $\left(\mathrm{P}_{H, q}\right)$ is -1 . Consequently, equation $\left(\mathrm{P}_{H, q}\right)$ with $q=3$ has at least one solution.

The hypothesis that $(M, g)$ is not conformally equivalent to the standard threedimensional ball is necessary since $\left(\mathrm{P}_{H}\right)$ may have no solution in this case due to the Kazdan-Warner-type condition for manifolds with boundary and for the mean curvature proved in [Escobar 1996]. On the ball sufficient conditions on $H$ in dimensions 3 and 4 are given in [Djadli et al. 2004; Escobar and Garcia 2004], and perturbative results were obtained in [Chang et al. 1998].

Recently S. Brendle [2002a; 2002b] obtained on surfaces some results related to ours. He used curvature flow methods, in the spirit of M. Struwe [2002] and X.-X. Chen [2001]. The curvature flow method was introduced in [Hamilton 1988] and used in [Chow 1991; Ye 1994; Bartz et al. 1994].

The remainder of the paper is organized as follows. In Section 2 we provide the main local blow-up analysis giving first sharp pointwise estimates to a sequence of solutions near isolated simple blow-up points, then we prove that an isolated blow-up is in fact an isolated simple blow-up, ruling out the possibility of bubbles on top of bubbles. In Section 3 we rule out the possibility of bubble accumulations and establish Theorem 1.1. In Section 4 we study compactness of solutions of $\left(\mathrm{P}_{H}\right)$ and establish Theorem 1.2. In the Appendix we provide some standard descriptions of singular behaviour of positive solutions to some linear boundary value elliptic equations in punctured half balls and collect some useful results. 


\section{Local blow-up analysis}

We may assume without loss of generality that $h_{g} \equiv 0$. Indeed, let $\varphi_{1}$ be a positive eigenfunction associated to the first eigenvalue $\lambda_{1}$ of the problem

$$
\begin{cases}L_{g} \varphi=\lambda_{1} \varphi & \text { in } \stackrel{\circ}{M}, \\ B_{g} \varphi=0 & \text { on } \partial M .\end{cases}
$$

Setting $\tilde{g}=\varphi_{1}^{4} g$ and $\tilde{u}=\varphi_{1}^{-1} u$, where $u$ is a solution of $\left(\mathrm{P}_{H, q}\right)$ (with $q=3$ ), one can easily check that $R_{\tilde{g}}>0, h_{\tilde{g}} \equiv 0$, and $\tilde{u}$ satisfies

$$
\begin{cases}L_{\tilde{g}} \tilde{u}=0 & \text { in } \stackrel{\circ}{\partial \tilde{u}}, \\ \frac{\partial \tilde{u}}{\partial v}=H \tilde{u}^{3} & \text { on } \partial M .\end{cases}
$$

For simplicity, we work with $\tilde{g}$, denoting it by $g$. Since $\partial M$ is umbilic with respect to $g$ and $h_{\tilde{g}}=0$, it follows that the second fundamental form vanishes at each point of the boundary, that is, the boundary is a totally geodesic submanifold. Hence we can take conformal normal coordinates around any point of the boundary [Escobar 1992b].

Recall the definitions of isolated and isolated simple blow-ups, first introduced by R. Schoen [1991] and used extensively by Y.-Y. Li [1995; 1996].

Definition 2.1 (isolated blow-up point). Let $(M, g)$ be a smooth compact $n$ dimensional Riemannian manifold with boundary and take $\bar{r}>0, \bar{c}>0, \bar{x} \in \partial M$. Let $H \in C^{0}\left(\overline{B_{\bar{r}}(\bar{x})}\right)$ be a positive function, where $B_{\bar{r}}(\bar{x})$ denotes the geodesic ball in $(M, g)$ of radius $\bar{r}$ centered at $\bar{x}$. Suppose that, for certain sequences $q_{i}=3-\tau_{i}$, $\tau_{i} \rightarrow 0, H_{i} \rightarrow H$ in $C^{2}\left(\overline{B_{\bar{r}}(\bar{x})}\right)$, the sequence $\left\{u_{i}\right\}_{i \in \mathbb{N}}$ solves

$$
\begin{cases}L_{g} u_{i}=0, \quad u_{i}>0 & \text { in } B_{\bar{r}}(\bar{x}), \\ \frac{\partial u_{i}}{\partial v}=H_{i} u_{i}^{q_{i}} & \text { on } \partial M \cap B_{\bar{r}}(\bar{x}) .\end{cases}
$$

We say that $\bar{x}$ is an isolated blow-up point of $\left\{u_{i}\right\}_{i}$ if there exists a sequence of local maximum points $x_{i}$ of $u_{i}$ such that $x_{i} \rightarrow \bar{x}, u_{i}\left(x_{i}\right) \rightarrow \infty$ and for some $C_{1}>0$,

$$
u_{i}(x) \leq C_{1} d\left(x, x_{i}\right)^{-1 /\left(q_{i}-1\right)}, \quad \text { for all } x \in B_{\bar{r}}\left(x_{i}\right) \text { and all } i .
$$

To describe the behaviour of blowing-up solutions near an isolated blow-up point, we define spherical averages of $u_{i}$ centered at $x_{i}$ as follows

$$
\bar{u}_{i}(r)=f_{M \cap \partial B_{r}(\bar{x})} u_{i}=\frac{1}{\operatorname{Vol}_{g}\left(M \cap \partial B_{r}(\bar{x})\right)} \int_{M \cap \partial B_{r}(\bar{x})} u_{i} .
$$

Definition 2.2 (isolated simple blow-up point). Let $x_{i} \rightarrow \bar{x}$ be an isolated blow-up point of $\left\{u_{i}\right\}_{i}$ as in Definition 2.1. We say that $x_{i} \rightarrow \bar{x}$ is an isolated simple blow-up 
point of $\left\{u_{i}\right\}_{i}$ if, for some positive constants $\tilde{r} \in(0, \bar{r})$ and $C_{2}>1$, the function $\bar{w}_{i}(r):=r^{1 /\left(q_{i}-1\right)} \bar{u}_{i}(r)$ satisfies, for large $i$,

$$
\bar{w}_{i}^{\prime}(r)<0 \text { for } r \text { satisfying } C_{2} u_{i}^{1-q_{i}}\left(x_{i}\right) \leq r \leq \tilde{r} .
$$

Notation. For later use we introduce the following symbols:

$\mathbb{R}_{+}^{3}$ is the open upper half-space $\left\{\left(x^{1}, x^{2}, x^{3}\right) \in \mathbb{R}^{2} \times \mathbb{R}: x^{3}>0\right\}$;

$B_{r}^{+}(\bar{x})$ is the open upper hemisphere $\left\{x=\left(x^{\prime}, x^{3}\right) \in \mathbb{R}_{+}^{3}:|x-\bar{x}|<r\right\}$;

$\bar{B}_{r}^{+}(\bar{x})$ is the closure of $B_{r}^{+}(\bar{x})$;

$\Gamma_{1}\left(B_{r}^{+}(\bar{x})\right)$ is the closed equatorial disk $\partial B_{r}^{+}(\bar{x}) \cap \partial \mathbb{R}_{+}^{3}$;

$\Gamma_{2}\left(B_{r}^{+}(\bar{x})\right)$ is the open upper hemisphere $\partial B_{r}(\bar{x}) \cap \mathbb{R}_{+}^{3}$;

$\bar{\Gamma}_{2}\left(B_{r}^{+}(\bar{x})\right)$ is the closure of $\Gamma_{2}\left(B_{r}^{+}(\bar{x})\right)$.

When the center of a ball is 0 we omit it from the notation, so $B_{r}^{+}=B_{r}^{+}(0)$, etc.

For any $\bar{x} \in \partial M$, by choosing a geodesic normal coordinate system centered at $\bar{x}$, we can assume without loss of generality that

$$
\begin{gathered}
\bar{x}=0, \quad g_{i j}(0)=\delta_{i j}, \quad B_{1}^{+} \subset M, \\
\left\{\left(x^{\prime}, 0\right)=\left(x^{1}, x^{2}, 0\right):\left|x^{\prime}\right|<1\right\} \subset \partial M, \quad \Gamma_{i j}^{k}(0)=0,
\end{gathered}
$$

where $\Gamma_{i j}^{k}$ is the Christoffel symbol.

Let $H_{i} \rightarrow H$ in $C^{2}\left(\Gamma_{1}\left(B_{3}^{+}\right)\right)$be a sequence of positive functions, $q_{i}$ a sequence of numbers satisfying $2 \leq q_{i} \leq 3$ and $q_{i} \rightarrow 3$, and $\left\{v_{i}\right\}_{i} \subset C^{2}\left(\bar{B}_{3}^{+}\right)$a sequence of solutions to

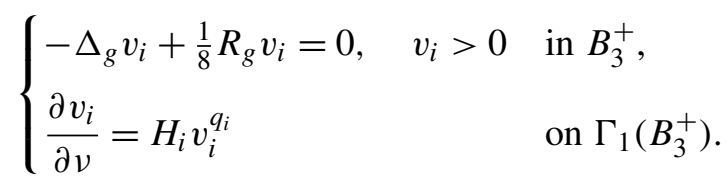

We now give some properties of isolated and isolated simple blow-ups. We will use $c$ to denote positive constants that may vary from formula to formula and may depend only on $M, g$, and $\bar{r}$. A similar analysis of blow-ups was also carried out in [Escobar and Garcia 2004], where $(M, g)$ was the standard ball endowed with euclidean metric. See also [Felli and Ould Ahmedou 2003].

The following lemma gives a Harnack Inequality, whose proof is contained (apart from minor modifications) in [Felli and Ould Ahmedou 2003, Lemma 2.3] and [Escobar and Garcia 2004].

Lemma 2.3. Let $v_{i}$ satisfy $\left(\mathrm{P}_{i}\right)$ and let $y_{i} \rightarrow \bar{y} \in \Gamma_{1}\left(B_{3}^{+}\right)$be an isolated blow-up of $\left\{v_{i}\right\}_{i}$. Then, for any $0<r<\bar{r}$,

$$
\max _{\bar{B}_{2 r}^{+}\left(y_{i}\right) \backslash B_{r / 2}^{+}\left(y_{i}\right)} v_{i} \leq C_{3} \min _{\bar{B}_{2 r}^{+}\left(y_{i}\right) \backslash B_{r / 2}^{+}\left(y_{i}\right)} v_{i},
$$

where $C_{3}$ is a positive constant independent of $i$ and $r$. 
Lemma 2.4. Let $v_{i}$ satisfy $\left(\mathrm{P}_{i}\right)$ and let $y_{i} \rightarrow \bar{y} \in \Gamma_{1}\left(B_{1}^{+}\right)$be an isolated blowup point. Then for any $R_{i} \rightarrow+\infty$ and $\varepsilon_{i} \rightarrow 0^{+}$we have, after passing to a subsequence,

$$
\begin{aligned}
& \left\|v_{i}^{-1}\left(y_{i}\right) v_{i}\left(\exp _{y_{i}}\left(v_{i}^{1-q_{i}}\left(y_{i}\right) x\right)\right)-\sqrt{\frac{1}{\left(1+h_{i} x^{3}\right)^{2}+h_{i}^{2}\left|x^{\prime}\right|^{2}}}\right\|_{C^{1}\left(B_{2 R_{i}}^{+}\right)} \\
& +\left\|v_{i}^{-1}\left(y_{i}\right) v_{i}\left(\exp _{y_{i}}\left(v_{i}^{1-q_{i}}\left(y_{i}\right) x\right)\right)-\sqrt{\frac{1}{\left(1+h_{i} x^{3}\right)^{2}+h_{i}^{2}\left|x^{\prime}\right|^{2}}}\right\|_{H^{1}\left(B_{2 R_{i}}^{+}\right)} \leq \varepsilon_{i}
\end{aligned}
$$

and

$$
\frac{R_{i}}{\log v_{i}\left(y_{i}\right)} \underset{i \rightarrow+\infty}{\longrightarrow} 0
$$

where $x=\left(x^{\prime}, x^{3}\right) \in B_{1}^{+}$and $h_{i}=H_{i}\left(y_{i}\right)$.

Proof. Let $g_{i}=\left(g_{i}\right)_{\alpha \beta}(x) d x^{\alpha} d x^{\beta}=g_{\alpha \beta}\left(v_{i}^{1-q_{i}}\left(y_{i}\right) x\right) d x^{\alpha} d x^{\beta}$ denote the scaled metric. Set

$$
\xi_{i}(x)=v_{i}^{-1}\left(y_{i}\right) v_{i}\left(y_{i}+v_{i}^{1-q_{i}}\left(y_{i}\right) x\right) \quad \text { for } x \in B_{v_{i}^{q_{i}-1}\left(y_{i}\right)}^{-T_{i}},
$$

defined on the set

$$
B_{v_{i}^{q_{i}-1}\left(y_{i}\right)}^{-T_{i}}:=\left\{z \in \mathbb{R}^{3}:|z|<v_{i}^{q_{i}-1}\left(y_{i}\right) \quad \text { and } \quad z^{3}>-T_{i}\right\},
$$

where $T_{i}=y_{i}^{3} v_{i}^{q_{i}-1}\left(y_{i}\right)$. Then the following conditions are satisfied:

(a) in $B_{v_{i}^{q_{i}-1}\left(y_{i}\right)}^{-T_{i}}$,

$$
-\Delta_{g_{i}} \xi_{i}+\frac{1}{8} v_{i}^{2\left(1-q_{i}\right)}\left(y_{i}\right) R_{g_{i}}\left(y_{i}+v_{i}^{1-q_{i}}\left(y_{i}\right) x\right) \xi_{i}=0 \quad \text { and } \quad \xi_{i}>0 ;
$$

(b) on $\partial B_{v_{i}^{q_{i}-1}\left(y_{i}\right)}^{-T_{i}} \cap\left\{z \in \mathbb{R}^{3}: z^{3}=-T_{i}\right\}$,

$$
\frac{\partial \xi_{i}}{\partial v_{g_{i}}}=H_{i}\left(y_{i}+v_{i}^{1-q_{i}}\left(y_{i}\right) x\right) \xi_{i}^{q_{i}}
$$

(c) $\xi_{i}(0)=1$,

(d) 0 is a local maximum point of $\xi_{i}$;

(e) for some positive constant $\tilde{c}$,

$$
0<\xi_{i}(x) \leq \tilde{c}|x|^{-1 /\left(q_{i}-1\right)} .
$$

Now we prove that $\xi_{i}$ is locally bounded. Using Hopf's boundary point lemma and Lemma 2.3, we derive that for $0<r<1$

$$
1=\xi_{i}(0) \geq \min _{\bar{\Gamma}_{1}\left(B_{r}^{+}\right)} \xi_{i} \geq \min _{\bar{\Gamma}_{2}\left(B_{r}^{+}\right)} \xi_{i} \geq c \max _{\bar{\Gamma}_{2}\left(B_{r}^{+}\right)} \xi_{i},
$$

which implies that, for some $c$ independent of $r$,

$$
\max _{\bar{\Gamma}_{2}\left(B_{r}^{+}\right)} \xi_{i} \leq c .
$$


From this we derive easily that $\xi_{i}$ is locally bounded. Applying standard elliptic estimates to $\left\{\xi_{i}\right\}$, we conclude, after passing to a subsequence, that $\xi_{i} \rightarrow \xi$ in $C_{\text {loc }}^{2}\left(\mathbb{R}_{+}^{3}\right)$ and $H_{\text {loc }}^{1}\left(\mathbb{R}_{+}^{3}\right)$ for some $\xi$ satisfying

$$
\begin{cases}\Delta \xi=0, \quad \xi>0 & \text { in } \mathbb{R}_{-T}^{3} \\ \frac{\partial \xi}{\partial v}=\left(\lim _{i} H_{i}\left(y_{i}\right)\right) \xi^{3} & \text { on } \partial \mathbb{R}_{-T}^{3},\end{cases}
$$

where $\mathbb{R}_{-T}^{3}:=\left\{x=\left(x^{\prime}, x^{3}\right) \in \mathbb{R}^{3}: x^{3}>-T\right\}$ and $T=\lim _{i} T_{i}$. By the Liouville Theorem and (2-4) we have $T<+\infty$. By a Liouville-type theorem from [Li and Zhu 1995] and [Chipot et al. 1996] (see Theorem A.3 in the Appendix), we easily deduce that $T=0$ and

$$
\xi\left(x^{\prime}, x^{3}\right)=\left(\frac{1}{\left(1+\lim _{i} H_{i}\left(y_{i}\right) x^{3}\right)^{2}+\left(\lim _{i} H_{i}\left(y_{i}\right)\right)^{2}\left|x^{\prime}\right|^{2}}\right)^{1 / 2} .
$$

Before stating our next result, we point out that it follows from Lemma A.5 of the Appendix that, for $\delta_{0}>0$ small enough, there exists a unique function $G(\cdot, \bar{y}) \in C^{2}\left(\bar{B}_{\delta_{o}}^{+}(\bar{y}) \backslash\{\bar{y}\}\right)$ satisfying

$$
\begin{cases}-\Delta_{g} G(\cdot, \bar{y})+\frac{1}{8} R_{g} G(\cdot, \bar{y})=0 & \text { in } B_{\delta_{o}}^{+}(\bar{y}), \\ \frac{\partial}{\partial v} G(\cdot, \bar{y})=0 & \text { on } \Gamma_{1}\left(B_{\delta_{o}}^{+}(\bar{y})\right) \backslash\{\bar{y}\}, \\ \lim _{y \rightarrow \bar{y}} d(y, \bar{y}) G(y, \bar{y})=1 . & \end{cases}
$$

Now we state our main estimate on isolated simple blow-up points.

Proposition 2.5. Let $v_{i}$ satisfy $\left(\mathrm{P}_{i}\right)$ and let $y_{i} \rightarrow \bar{y} \in \Gamma_{1}\left(B_{1}^{+}\right)$be an isolated simple blow-up point, with (2-2) and (2-3) for all $i$. Then for some positive constant $C$ depending only on $C_{1}, \tilde{r},\left\|H_{i}\right\|_{C^{2}\left(\Gamma_{1}\left(B_{3}^{+}\right)\right)}$, and $\inf _{y \in \Gamma_{1}\left(B_{1}^{+}\right)} H_{i}(y)$ we have

$$
v_{i}(y) \leq C v_{i}^{-1}\left(y_{i}\right) d\left(y, y_{i}\right)^{-1}, \quad \text { for } \quad d\left(y, y_{i}\right) \leq \frac{\tilde{r}}{2}
$$

where $C_{1}$ and $\tilde{r}$ are given in Definitions 2.1 and 2.2. Furthermore, after passing to some subsequence, for some positive constant $b$,

$$
v_{i}\left(y_{i}\right) v_{i} \underset{i \rightarrow+\infty}{\longrightarrow} b G(\cdot, \bar{y})+E \quad \text { in } C_{\mathrm{loc}}^{2}\left(\bar{B}_{\tilde{\rho}}^{+}(\bar{y}) \backslash\{\bar{y}\}\right),
$$

where $\tilde{\rho}=\min \left(\delta_{0}, \tilde{r} / 2\right)$ and $E \in C^{2}\left(B_{\tilde{\rho}}^{+}(\bar{y})\right)$ satisfies

$$
\begin{cases}-\Delta_{g} E+\frac{1}{8} R_{g} E=0 & \text { in } B_{\tilde{\rho}}^{+}, \\ \frac{\partial E}{\partial v}=0 & \text { on } \Gamma_{1}\left(B_{\tilde{\rho}}^{+}\right) .\end{cases}
$$

Proposition 2.5 will be established through a series of lemmas. 
Lemma 2.6. Let $v_{i}$ satisfy $\left(\mathrm{P}_{i}\right)$ and let $y_{i} \rightarrow \bar{y} \in \Gamma_{1}\left(B_{1}^{+}\right)$be an isolated simple blow-up. Assume $R_{i} \rightarrow+\infty$ and $0<\varepsilon_{i}<e^{-R_{i}}$ are sequences for which (2-2) and (2-3) hold. Then, for any $0<\delta<\frac{1}{100}$, there exists $\rho_{1} \in(0, \tilde{r})$ independent of $i$ (but depending on $\delta$ ) such that

$$
\begin{aligned}
& v_{i}\left(y_{i}\right) \leq C_{4} v_{i}^{-\lambda_{i}}\left(y_{i}\right) d\left(y, y_{i}\right)^{-1+\delta} \quad \text { for all } r_{i} \leq d\left(y, y_{i}\right) \leq \rho_{1}, \\
& \nabla_{g} v_{i}\left(y_{i}\right) \leq C_{4} v_{i}^{-\lambda_{i}}\left(y_{i}\right) d\left(y, y_{i}\right)^{-2+\delta} \quad \text { for all } r_{i} \leq d\left(y, y_{i}\right) \leq \rho_{1} \text {, } \\
& \nabla_{g}^{2} v_{i}\left(y_{i}\right) \leq C_{4} v_{i}^{-\lambda_{i}}\left(y_{i}\right) d\left(y, y_{i}\right)^{-3+\delta} \quad \text { for all } r_{i} \leq d\left(y, y_{i}\right) \leq \rho_{1} \text {, }
\end{aligned}
$$

where $r_{i}=R_{i} v_{i}^{1-q_{i}}\left(y_{i}\right), \lambda_{i}=(1-\delta)\left(q_{i}-1\right)-1$, and $C_{4}$ is some positive constant independent of $i$.

Proof. We assume for simplicity that $g$ is the flat metric. The general case can be derived essentially in the same way. Let $r_{i}=R_{i} v_{i}^{1-q_{i}}\left(y_{i}\right)$. Lemma 2.4 implies that

$$
v_{i}(y) \leq c v_{i}\left(y_{i}\right) R_{i}^{-1} \quad \text { for } d\left(y, y_{i}\right)=r_{i} .
$$

We then derive from Lemma 2.3, (2-9), and the definition of an isolated simple blow-up that, for $r_{i} \leq d\left(y, y_{i}\right) \leq \tilde{r}$, we have

$$
v_{i}^{q_{i}-1}(y) \leq c R_{i}^{-1+o(1)} d\left(y, y_{i}\right)^{-1} .
$$

Set $T_{i}=y_{i}^{3} v_{i}^{q_{i}-1}\left(y_{i}\right)$. From the proof of Lemma 2.4 we know that $\lim _{i} T_{i}=0$. It is not restrictive to take $y_{i}=\left(0,0, y_{i}^{3}\right)$. Thus we have $d\left(0, y_{i}^{3}\right)=o\left(r_{i}\right)$. So

$$
B_{1}^{+}(0) \backslash B_{2 r_{i}}^{+}(0) \subset\left\{\frac{3}{2} r_{i} \leq d\left(y, y_{i}\right) \leq \frac{3}{2}\right\} .
$$

We now apply the maximum principle stated in Theorem A.1; to this aim we set

$$
\varphi_{i}(y)=M_{i}\left(|y|^{-\delta}-\varepsilon|y|^{\delta-1} y^{3}\right)+A v_{i}^{-\lambda_{i}}\left(y_{i}\right)\left(|y|^{-1+\delta}-\varepsilon|y|^{-2+\delta} y^{3}\right)
$$

with $M_{i}$ and $A$ to be chosen later, and let $\Phi_{i}$ be the boundary operator defined by

$$
\Phi_{i}(v)=\frac{\partial v}{\partial v}-H_{i} v_{i}^{q_{i}-1}\left(y_{i}\right) v
$$

A direct computation yields

$$
\Delta \varphi_{i}(y)=M_{i}|y|^{-\delta}(-\delta(1-\delta)+O(\varepsilon))+|y|^{-(3-\delta)} A v_{i}^{-\lambda_{i}}\left(y_{i}\right)(-\delta(1-\delta)+O(\varepsilon)) .
$$

Thus one can choose $\varepsilon=O(\delta)$ such that $\Delta \varphi_{i} \leq 0$.

Another straightforward computation taking into account (2-10) shows that for $\delta>0$ there exists $\rho_{1}(\delta)>0$ such that

$$
\Phi_{i} \varphi_{i}>0 \quad \text { on } \Gamma_{1}\left(B_{\rho_{1}}^{+}\right) .
$$

Setting

$$
\begin{array}{lll}
\Omega=D_{i}=B_{\rho_{1}}^{+} \backslash B_{2 r_{i}}^{+}(0), & \\
\Sigma=\Gamma_{1}\left(D_{i}\right):=\partial D_{i} \cap \partial \mathbb{R}_{+}^{3}, & \Gamma=\Gamma_{2}\left(D_{i}\right):=\partial D_{i} \cap \mathbb{R}_{+}^{3}, & V \equiv 0, \\
h=H_{i} v_{i}^{q_{i}-1}, & v=\varphi_{i}-v_{i}, & \psi=v_{i},
\end{array}
$$


and choosing $A=O(\delta)$ such that $\varphi_{i} \geq 0$ on $\Gamma_{2}\left(D_{i}\right)$ and $M_{i}=\max _{\Gamma_{1}\left(B_{\rho_{1}}^{+}\right)} v_{i}$, we deduce from Theorem A.1 that

$$
v_{i}(x) \leq \varphi_{i}(x) .
$$

By the Harnack inequality and the assumption that the blow-up is isolated simple, we derive that

$$
M_{i} \leq c v_{i}^{-\lambda_{i}}\left(y_{i}\right) .
$$

Now (2-6) follows from (2-11) and (2-12).

To derive (2-7) from (2-6), we argue as follows. For $r_{i} \leq|\tilde{y}| \leq \rho_{1} / 2$, we consider

$$
w_{i}(z)=|\tilde{y}|^{1-\delta} v_{i}^{\lambda_{i}}\left(y_{i}\right) v_{i}(|\tilde{y}| z) \quad \text { for } \frac{1}{2} \leq|z| \leq 2, z^{3} \geq 0 .
$$

It follows from $\left(\mathrm{P}_{i}\right)$ that $w_{i}$ satisfies

$$
\begin{cases}-\Delta w_{i}=0 & \text { in }\left\{\frac{1}{2}<|z|<2: z^{3}>0\right\}, \\ \frac{\partial w_{i}}{\partial v}=H_{i}(|\tilde{y}| z)|\tilde{y}|^{-\lambda_{i}} v_{i}^{\lambda_{i}\left(1-q_{i}\right)}\left(y_{i}\right) w_{i}^{q_{i}} & \text { on }\left\{\frac{1}{2}<|z|<2: z^{3}=0\right\} .\end{cases}
$$

In view of (2-6), we have $w_{i}(z) \leq c$ for any $z$ such that $\frac{1}{2} \leq|z| \leq 2$ and $z^{3} \geq 0$. We then derive from (2-13) and gradient elliptic estimates that

$$
\left|\nabla w_{i}(z)\right| \leq c \quad \text { for } z \in \Gamma_{2}\left(B_{1}^{+}\right),
$$

which implies that

$$
\left|\nabla v_{i}(\tilde{y})\right| \leq c|\tilde{y}|^{-2+\delta} v_{i}^{-\lambda_{i}}\left(y_{i}\right) .
$$

This establishes (2-7). Estimate (2-8) can be derived in a similar way. We omit the details.

Later on we will fix $\delta$ close to 0 , hence fix $\rho_{1}$. Our aim is to obtain (2-6) with $\delta=0$ for $r_{i} \leq d\left(y, y_{i}\right) \leq \rho_{1}$, which together with Lemma 2.4 yields Proposition 2.5.

Now we state a Pohozaev-type identity, which is basically contained in [Li and Zhu 1997]. In the following, we will be working in some geodesic normal coordinate $x=\left(x^{1}, x^{2}, x^{3}\right)$ with $g_{i j}(0)=\delta_{i j}$ and $\Gamma_{i j}^{k}(0)=0$. We use also the notation $\nabla=\left(\partial_{1}, \partial_{2}, \partial_{3}\right), d x=d x^{1} \wedge d x^{2} \wedge d x^{3}$ and $d s$ to denote the surface area element with respect to the flat metric.

Lemma 2.7. For $H \in C^{2}\left(\Gamma_{1}\left(B_{1}^{+}\right)\right)$and $a \in C^{2}\left(\Gamma_{1}\left(B_{1}^{+}\right)\right)$, let $u \in C^{2}\left(\bar{B}_{1}^{+}\right)$satisfy, for $q>0$,

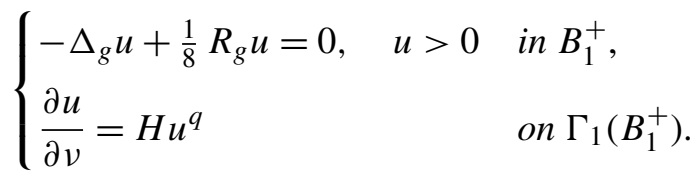


Then, for any $r$ such that $0<r \leq 1$,

$$
\begin{aligned}
& \frac{1}{q+1} \int_{\Gamma_{1}\left(B_{r}^{+}\right)}\left(x^{\prime} \cdot \nabla_{x^{\prime}} H\right) u^{q+1} d s+\left(\frac{2}{q+1}-\frac{1}{2}\right) \int_{\Gamma_{1}\left(B_{r}^{+}\right)} H u^{q+1} d s \\
& \quad-\frac{1}{16} \int_{B_{r}^{+}}\left(x \cdot \nabla R_{g}\right) u^{2} d x-\frac{1}{8} \int_{B_{r}^{+}} R_{g} u^{2} d x-\frac{r}{16} \int_{\Gamma_{2}\left(B_{r}^{+}\right)} R_{g} u^{2} d s \\
& \quad-\frac{r}{q+1} \int_{\partial \Gamma_{1}\left(B_{r}^{+}\right)} H u^{q+1} d s \\
& =\int_{\Gamma_{2}\left(B_{r}^{+}\right)} B(r, x, u, \nabla u) d s+A(g, u),
\end{aligned}
$$

where

$$
B(r, x, u, \nabla u)=\frac{1}{2} u \frac{\partial u}{\partial v}+\frac{1}{2} r\left(\frac{\partial u}{\partial v}\right)^{2}-\frac{1}{2} r\left|\nabla_{T} u\right|^{2}
$$

$\left(\nabla_{T} u\right.$ being the component of $\nabla u$ tangent to $\left.\Gamma_{2}\left(B_{r}^{+}\right)\right)$and

$$
\begin{aligned}
A(g, u)=\int_{B_{r}^{+}}\left(x^{k} \partial_{k} u\right)\left(g_{i j}-\delta_{i j}\right) \partial_{i j} u d x-\int_{B_{r}^{+}}\left(x^{l} \partial_{l} u\right)\left(g_{i j}-\Gamma_{i j}^{k} \partial_{k} u\right) d x \\
\quad+\frac{1}{2} \int_{B_{r}^{+}} u\left(g^{i j}-\delta^{i j}\right) \partial_{i j} u d x-\frac{1}{2} \int_{B_{r}^{+}} u g^{i j} \Gamma_{i j}^{k} \partial_{k} u d x \\
\quad-\int_{\Gamma_{1}\left(B_{r}^{+}\right)} x^{i} \frac{\partial u}{\partial x_{i}}\left(g^{i j}-\delta^{i j}\right) \frac{\partial u}{\partial x_{i}} v_{j}-\frac{n-2}{2} \int_{\Gamma_{1}\left(B_{r}^{+}\right)}\left(g^{i j}-\delta^{i j}\right) \frac{\partial u}{\partial x_{i}} v_{j} u
\end{aligned}
$$

Regarding the term $A\left(g, u_{i}\right)$, where $u_{i}$ is a solution of $\left(\mathrm{P}_{i}\right)$, we have the following estimate, whose proof is a direct consequence of Lemmas 2.4 and 2.6.

Lemma 2.8. Let $\left\{v_{i}\right\}_{i}$ satisfy $\left(\mathrm{P}_{i}\right)$ and let $y_{i} \rightarrow \bar{y} \in \Gamma_{1}\left(B_{1}^{+}\right)$be an isolated simple blow-up point. Assume that $R_{i} \rightarrow+\infty$ and $0<\varepsilon_{i}<e^{-R_{i}}$ are sequences for which (2-2) and (2-3) hold. Then, for $0<r<\rho_{1}$, we have

$$
\left|A\left(g, v_{i}\right)\right| \leq C_{5} r v_{i}^{-2 \lambda_{i}}\left(y_{i}\right),
$$

where $C_{5}$ is some constant independent of $i$ and $r$.

Using Lemmas 2.4, 2.6, 2.7 and 2.8, together with standard elliptic estimates, we derive the following estimate about the rate of blow-up of the solutions of $\left(\mathrm{P}_{i}\right)$.

Lemma 2.9. Let $v_{i}$ satisfy $\left(\mathrm{P}_{i}\right)$ and let $y_{i} \rightarrow \bar{y} \in \Gamma_{1}\left(B_{1}^{+}\right)$be an isolated simple blow-up point. Assume that $R_{i} \rightarrow+\infty$ and $0<\varepsilon_{i}<e^{-R_{i}}$ are sequences for which (2-2) and (2-3) hold. Then

$$
\tau_{i}=O\left(v_{i}^{-2 \lambda_{i}}\left(y_{i}\right)\right) .
$$

Consequently $v_{i}^{\tau_{i}}\left(y_{i}\right) \rightarrow 1$ as $i \rightarrow \infty$. 
Lemma 2.10. Let $v_{i}$ satisfy $\left(\mathrm{P}_{i}\right)$ and let $y_{i} \rightarrow \bar{y} \in \Gamma_{1}\left(B_{1}^{+}\right)$be an isolated simple blow-up point. Then, for $0<r<\tilde{r} / 2$, we have

$$
\limsup _{i \rightarrow+\infty} \max _{y \in \Gamma_{2}\left(B_{r}^{+}\left(y_{i}\right)\right)} v_{i}\left(y_{i}\right) v_{i}(y) \leq C(r) .
$$

Proof. By Lemma 2.3, it is enough to establish the lemma for $r>0$ small enough. Without loss of generality we may take $\bar{r}=1$. Pick any $y_{r} \in \Gamma_{2}\left(B_{r}^{+}\right)$and set

$$
\xi_{i}(y)=v_{i}^{-1}\left(y_{r}\right) v_{i}(y) \text {. }
$$

Then $\xi_{i}$ satisfies

$$
\begin{cases}-\Delta_{g} \xi_{i}+\frac{1}{8} R_{g} \xi_{i}=0 & \text { in } B_{1 / 2}^{+}(\bar{y}), \\ \frac{\partial \xi_{i}}{\partial v}=H_{i} v_{i}^{q_{i}-1}\left(y_{r}\right) \xi_{i}^{q_{i}} & \text { on } \Gamma_{1}\left(B_{1 / 2}^{+}(\bar{y})\right) .\end{cases}
$$

It follows from Lemma 2.3 that for any compact set $K \subset B_{1 / 2}^{+}(\bar{y}) \backslash\{\bar{y}\}$ there exists some constant $c(K)$ such that

$$
c(K)^{-1} \leq \xi_{i} \leq c(K) \quad \text { on } K .
$$

We also know from (2-6) that $v_{i}\left(y_{r}\right) \rightarrow 0$ as $i \rightarrow+\infty$. Then by standard elliptic theory, we have, after passing to a subsequence, that $\xi_{i} \rightarrow \xi$ in $C_{\mathrm{loc}}^{2}\left(B_{1 / 2}^{+}(\bar{y}) \backslash\{\bar{y}\}\right)$, where $\xi$ satisfies

$$
\begin{cases}-\Delta_{g} \xi+\frac{1}{8} R_{g} \xi=0 & \text { in } B_{1 / 2}^{+}(\bar{y}), \\ \frac{\partial \xi}{\partial v}=0 & \text { on } \Gamma_{1}\left(B_{1 / 2}^{+}\right) \backslash\{\bar{y}\} .\end{cases}
$$

From the assumption that $y_{i} \rightarrow \bar{y}$ is an isolated simple blow-up point of $\left\{v_{i}\right\}_{i}$, we know that the function $r^{1 / 2} \bar{\xi}(r)$ is nonincreasing in the interval $(0, \tilde{r})$ and so we deduce that $\xi$ is singular at $\bar{y}$. So it follows from Corollary A.8 that for $r$ small enough there exists some positive constant $m>0$ independent of $i$ such that for $i$ large we have

$$
-\frac{1}{8} \int_{B_{r}^{+}} R_{g} \xi_{i}=\int_{B_{r}^{+}}-\Delta_{g} \xi_{i}=-\int_{\Gamma_{1}\left(B_{r}^{+}\right)} \frac{\partial \xi_{i}}{\partial v}-\int_{\Gamma_{2}\left(B_{r}^{+}\right)} \frac{\partial \xi_{i}}{\partial v}>m-\int_{\Gamma_{1}\left(B_{r}^{+}\right)} \frac{\partial \xi_{i}}{\partial v},
$$

which implies that

$$
-\frac{1}{8} \int_{B_{r}^{+}} R_{g} \xi_{i}+\int_{\Gamma_{1}\left(B_{r}^{+}\right)} \frac{\partial \xi_{i}}{\partial v}>m
$$

On the other hand,

$$
\int_{\Gamma_{1}\left(B_{r}^{+}\right)} \frac{\partial \xi_{i}}{\partial v}=\int_{\Gamma_{1}\left(B_{r}^{+}\right)} H_{i} v_{i}^{q_{i}-1}\left(y_{r}\right) \xi_{i}^{q_{i}} \leq v_{i}^{-1}\left(y_{r}\right) \int_{\Gamma_{1}\left(B_{r}^{+}\right)} H_{i} v_{i}^{q_{i}} .
$$

Using Lemmas 2.4 and 2.6, we derive that

$$
\int_{\Gamma_{1}\left(B_{r}^{+}\right)} H_{i} v_{i}^{q_{i}} \leq c v_{i}^{-1}\left(y_{i}\right)
$$


Hence our lemma follows from (2-16), (2-17), and (2-18).

Proof of Proposition 2.5. We first establish (2-5) arguing by contradiction. Suppose the contrary; then, possibly passing to a subsequence still denoted by $v_{i}$, there exists a sequence $\left\{\tilde{y}_{i}\right\}_{i}$ such that $d\left(\tilde{y}_{i}, y_{i}\right) \leq \tilde{r} / 2$ and

$$
v_{i}\left(\tilde{y}_{i}\right) v_{i}\left(y_{i}\right) d\left(\tilde{y}_{i}, y_{i}\right) \underset{i \rightarrow+\infty}{\longrightarrow}+\infty .
$$

Set $\tilde{r}_{i}=d\left(\tilde{y}_{i}, y_{i}\right)$. From Lemma 2.4 it is clear that $\tilde{r}_{i} \geq r_{i}=R_{i} v_{i}^{1-q_{i}}\left(y_{i}\right)$. Set

$$
\tilde{v}_{i}(x)=\tilde{r}_{i}^{1 /\left(q_{i}-1\right)} v_{i}\left(y_{i}+\tilde{r}_{i} x\right) \quad \text { in } B_{2}^{-T_{i}}, \quad T_{i}=\tilde{r}_{i}^{-1} y_{i}^{3} .
$$

Clearly $\tilde{v}_{i}$ satisfies

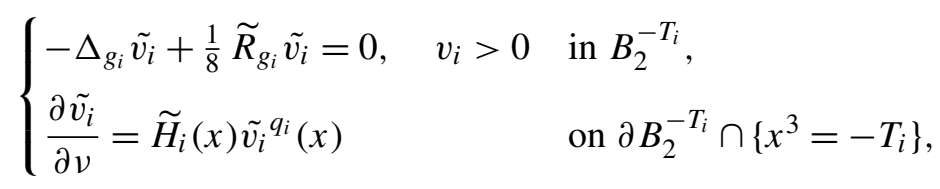

where

$$
\begin{aligned}
\left(g_{i}\right)_{\alpha \beta} & =g_{\alpha \beta}\left(\tilde{r}_{i} x\right) d x^{\alpha} d x^{\beta}, \\
\widetilde{R}_{g_{i}}(x) & =\tilde{r}_{i}^{2} R_{g_{i}}\left(y_{i}+\tilde{r}_{i} x\right), \\
\widetilde{H}_{i}(x) & =H_{i}\left(y_{i}+\tilde{r}_{i} x\right) .
\end{aligned}
$$

Lemma 2.10 yields $\max _{x \in \Gamma_{2}\left(B_{1 / 2}^{+}\right)} \tilde{v}_{i}(0) \tilde{v}_{i}(x) \leq c$ for some positive constant $c$, so

$$
v_{i}\left(\tilde{y}_{i}\right) v_{i}\left(y_{i}\right) d\left(y_{i}, y_{i}\right) \leq c .
$$

This contradicts (2-19). Therefore (2-5) is established. Now take

$$
w_{i}(x)=v_{i}\left(y_{i}\right) v_{i}(x) .
$$

From $\left(\mathrm{P}_{i}\right)$ it is clear that $w_{i}$ satisfies

$$
\begin{cases}-\Delta_{g} w_{i}+\frac{1}{8} R_{g} w_{i}=0, & \text { in } B_{3}^{+} \\ \frac{\partial w_{i}}{\partial v}=H_{i}(x) v_{i}^{1-q_{i}}\left(y_{i}\right) w_{i}^{q_{i}} & \text { on } \Gamma_{1}\left(B_{3}^{+}\right)\end{cases}
$$

Estimate (2-5) implies that $w_{i}(x) \leq c d\left(x, y_{i}\right)^{-1}$. Since $y_{i} \rightarrow \bar{y}, w_{i}$ is locally bounded in any compact set not containing $\bar{y}$. Then, up to a subsequence, $w_{i} \rightarrow w$ in $C_{\text {loc }}^{2}\left(B_{\tilde{\rho}}(\bar{y}) \backslash\{\bar{y}\}\right)$ for some $w>0$ satisfying

$$
\begin{cases}-\Delta_{g} w+\frac{1}{8} R_{g} w=0 & \text { in } B_{\tilde{\rho}}^{+}(\bar{y}) \\ \frac{\partial w}{\partial v}=0 & \text { on } \Gamma_{1}\left(B_{\tilde{\rho}}^{+}\right) \backslash\{\bar{y}\} .\end{cases}
$$

From Proposition A.7, we have

$$
w=b G(\cdot, \bar{y})+E \quad \text { in } B_{\tilde{\rho}}^{+} \backslash\{0\},
$$


where $b \geq 0, E$ is a regular function satisfying

$$
\begin{cases}-\Delta_{g} E+\frac{1}{8} R_{g} E=0 & \text { in } B_{\tilde{\rho}}^{+}, \\ \frac{\partial E}{\partial v}=0 & \text { on } \Gamma_{1}\left(B_{\tilde{\rho}}^{+}\right),\end{cases}
$$

and $G \in C^{2}\left(B_{\tilde{\rho}}^{+} \backslash\{\bar{y}\}\right)$ satisfies

$$
\begin{cases}-L_{g} G(\cdot, \bar{y})=0 & \text { in } B_{\tilde{\rho}}^{+}, \\ \frac{\partial G_{a}}{\partial v}=0 & \text { on } \Gamma_{1}\left(B_{\tilde{\rho}}^{+}\right) \backslash\{\bar{y}\},\end{cases}
$$

and $\lim _{y \rightarrow \bar{y}} d(y, \bar{y}) G(y, \bar{y})$ is a constant. Moreover $w$ is singular at $\bar{y}$. Indeed, from the definition of an isolated simple blow-up we know that $r^{1 / 2} \bar{w}(r)$ is a nonincreasing function in the interval $(0, \tilde{r})$, which implies that $w$ is singular at the origin and hence $b>0$. The proof of Proposition 2.5 is thereby complete.

Using Proposition 2.5, one can strengthen the results of Lemmas 2.6 and 2.8 using just (2-5) instead of (2-6), thus obtaining the following corollary.

Corollary 2.11. Let $\left\{v_{i}\right\}_{i}$ satisfy $\left(\mathrm{P}_{i}\right)$ and let $y_{i} \rightarrow \bar{y} \in \Gamma_{1}\left(B_{1}^{+}\right)$be an isolated simple blow-up point. Assume that $R_{i} \rightarrow+\infty$ and $0<\varepsilon_{i}<e^{-R_{i}}$ are sequences for which (2-2) and (2-3) hold. Then there exists $\rho_{1} \in(0, \tilde{r})$ such that

$$
\left|\nabla_{g} v_{i}(y)\right| \leq C_{4} v_{i}^{-1}\left(y_{i}\right) d\left(y, y_{i}\right)^{-2} \text { for all } r_{i} \leq d\left(y, y_{i}\right) \leq \rho_{1}
$$

and

$$
\left|\nabla_{g}^{2} v_{i}(y)\right| \leq C_{4} v_{i}^{-1}\left(y_{i}\right) d\left(y, y_{i}\right)^{-3} \quad \text { for all } r_{i} \leq d\left(y, y_{i}\right) \leq \rho_{1},
$$

where $r_{i}=R_{i} v_{i}^{1-q_{i}}\left(y_{i}\right)$ and $C_{4}$ is some positive constant independent of $i$. Moreover

$$
\left|A\left(g, v_{i}\right)\right| \leq C_{5} r v_{i}^{-2}\left(y_{i}\right),
$$

for some positive constant $C_{5}$ independent of $i$.

We prove an upper bound estimate for $\nabla_{g} H_{i}\left(y_{i}\right)$.

Lemma 2.12. Let $v_{i}$ satisfy $\left(\mathrm{P}_{i}\right)$ and let $y_{i} \rightarrow \bar{y} \in \Gamma_{1}\left(B_{1}^{+}\right)$be an isolated simple blow-up point. Then

$$
\nabla_{g} H_{i}\left(y_{i}\right)=O\left(v_{i}^{-2}\left(y_{i}\right)\right) .
$$

Proof. Let $x=\left(x^{1}, x^{2}, x^{3}\right)$ be geodesic normal coordinates centered at $y_{i}$ and let $\eta$ be a smooth cut-off function such that $0 \leq \eta \leq 1$ and

$$
\eta(x)= \begin{cases}1 & \text { if } x \in \bar{B}_{1 / 4}^{+}, \\ 0 & \text { if } x \notin \bar{B}_{1 / 2}^{+} .\end{cases}
$$


Multiply $\left(\mathrm{P}_{i}\right)$ by $\eta\left(\partial v_{i} / \partial x_{1}\right)$ and integrate by parts over $B_{1}^{+}$, thus obtaining

$$
0=\int_{B_{1}^{+}} \nabla_{g} v_{i} \cdot \nabla\left(\eta \frac{\partial v_{i}}{\partial x_{1}}\right) d V+\frac{1}{8} \int_{B_{1}^{+}} R_{g} v_{i} \eta \frac{\partial v_{i}}{\partial x_{1}}-\int_{\Gamma_{1}\left(B_{1 / 2}^{+}\right)} \frac{\partial v_{i}}{\partial v} \eta \frac{\partial v_{i}}{\partial x_{1}} d \sigma
$$

From $\left(\mathrm{P}_{i}\right),(2-5)$, and (2-2) we have

$$
\begin{aligned}
\int_{\Gamma_{1}\left(B_{1 / 2}^{+}\right)} \frac{\partial v_{i}}{\partial v} \eta \frac{\partial v_{i}}{\partial x_{1}} d \sigma+\frac{1}{8} \int_{B_{1}^{+}} R_{g} v_{i} \eta \frac{\partial v_{i}}{\partial x_{1}} \\
=\int_{\Gamma_{1}\left(B_{1 / 2}^{+}\right)} H_{i} v_{i}^{q_{i}} \eta \frac{\partial v_{i}}{\partial x_{1}} d \sigma+O\left(v_{i}^{-2}\left(y_{i}\right)\right) \\
=-\frac{1}{q_{i}+1} \frac{\partial H_{i}}{\partial x_{1}}\left(y_{i}\right) \int_{\Gamma_{1}\left(B_{1 / 2}^{+}\right)} \eta v_{i}^{q_{i}+1} d \sigma+O\left(\int_{\Gamma_{1}\left(B_{1 / 2}^{+}\right)}\left|x^{\prime}\right| v_{i}^{q_{i}+1}\right)+O\left(v_{i}^{-2}\left(y_{i}\right)\right) \\
=-\frac{1}{q_{i}+1} \frac{\partial H_{i}}{\partial x_{1}}\left(y_{i}\right) \int_{\Gamma_{1}\left(B_{1 / 2}^{+}\right)} \eta v_{i}^{q_{i}+1} d \sigma+O\left(v_{i}^{-2}\left(y_{i}\right)\right) .
\end{aligned}
$$

On the other hand, from (2-20) it follows that

$$
\begin{aligned}
\int_{\Gamma_{1}\left(B_{1}^{+}\right)} \nabla_{g} & v_{i} \\
& \nabla_{g}\left(\eta \frac{\partial v_{i}}{\partial x_{1}}\right) d \sigma \\
& =\int_{B_{1}^{+}}\left(\nabla_{g} v_{i} \cdot \nabla_{g} \eta\right) \frac{\partial v_{i}}{\partial x_{1}} d V+\int_{B_{1}^{+}} \nabla_{g} v_{i} \cdot \eta \nabla_{g}\left(\frac{\partial v_{i}}{\partial x_{1}}\right) d V \\
& =-\frac{1}{2} \int_{B_{1 / 2}^{+} \backslash B_{1 / 4}^{+}} \frac{\partial \eta}{\partial x_{1}}\left|\nabla_{g} v_{i}\right|^{2} d V+O\left(v_{i}^{-2}\left(y_{i}\right)\right)=O\left(v_{i}^{-2}\left(y_{i}\right)\right) .
\end{aligned}
$$

Putting together (2-22), (2-23), and (2-24), we find

$$
\frac{\partial H_{i}}{\partial x_{1}}\left(y_{i}\right)=O\left(v_{i}^{-2}\left(y_{i}\right)\right) \text {. }
$$

Repeating the same argument for the derivatives with respect to $x_{2}$ and $x_{3}$, we come to the required estimate.

Corollary 2.13. Under the assumptions of Lemma 2.12,

$$
\int_{\Gamma_{1}\left(B_{r}^{+}\right)} x^{\prime} \cdot \nabla_{x^{\prime}} H_{i} v_{i}^{q_{i}+1} d \sigma=O\left(v_{i}^{-4}\left(y_{i}\right)\right) .
$$

Proof. We have

$$
\begin{aligned}
\int_{\Gamma_{1}\left(B_{r}^{+}\right)} x^{\prime} \cdot \nabla_{x^{\prime}} H_{i} v_{i}^{q_{i}+1} d \sigma \\
=\int_{\Gamma_{1}\left(B_{r}^{+}\right)} \nabla_{x^{\prime}} H_{i}\left(y_{i}\right) \cdot\left(x^{\prime}-y_{i}\right) v_{i}^{q_{i}+1} d \sigma+O\left(\int_{\Gamma_{1}\left(B_{r}^{+}\right)}\left|x^{\prime}\right|^{2} v_{i}^{q_{i}+1} d \sigma\right) .
\end{aligned}
$$

From Proposition 2.5 and Lemma 2.4, $\int_{\Gamma_{1}\left(B_{r}^{+}\right)}\left(x^{\prime}-y_{i}\right) v_{i}^{q_{i}+1} d \sigma=O\left(v_{i}^{-2}\left(y_{i}\right)\right)$. The conclusion follows from Lemma 2.12, Corollary 2.11, and (2-2). 
Proposition 2.14. Let $v_{i}$ satisfy $\left(\mathrm{P}_{i}\right), y_{i} \rightarrow \bar{y}$ be an isolated simple blow-up point with, for some $\tilde{\rho}>0$,

$$
v_{i}\left(y_{i}\right) v_{i} \underset{i \rightarrow+\infty}{\longrightarrow} h \text { in } C_{\mathrm{loc}}^{2}\left(B_{\tilde{\rho}}^{+}(\bar{y}) \backslash\{\bar{y}\}\right) .
$$

Assume, for some $\beta>0$, that in some geodesic normal coordinate system $x=$ $\left(x^{1}, x^{2}, x^{3}\right)$ we have

$$
h(x)=\frac{\beta}{|x|}+A+o(1) \quad \text { as }|x| \rightarrow 0 .
$$

Then $A \leq 0$.

Proof. For $r>0$ small, the Pohozaev-type identity of Lemma 2.7 yields

$$
\begin{aligned}
\frac{1}{q_{i}+1} & \int_{\Gamma_{1}\left(B_{r}^{+}\right)}\left(x^{\prime} \cdot \nabla_{x^{\prime}} H_{i}\right) v_{i}^{q_{i}+1} d s+\left(\frac{2}{q_{i}+1}-\frac{1}{2}\right) \int_{\Gamma_{1}\left(B_{r}^{+}\right)} H_{i} v_{i}^{q_{i}+1} d s \\
& \quad-\frac{1}{16} \int_{B_{r}^{+}}\left(x \cdot \nabla R_{g}\right) v_{i}^{2} d x-\frac{1}{8} \int_{B_{r}^{+}} R_{g} v_{i}^{2} d x-\frac{r}{16} \int_{\Gamma_{2}\left(B_{r}^{+}\right)} R_{g} v_{i}^{2} d s \\
& \quad-\frac{r}{q_{i}+1} \int_{\partial \Gamma_{1}\left(B_{r}^{+}\right)} H_{i} v_{i}^{q_{i}+1} \\
= & \int_{\Gamma_{2}\left(B_{r}^{+}\right)} B\left(r, x, v_{i}, \nabla v_{i}\right) d s+A\left(g, v_{i}\right),
\end{aligned}
$$

where $B$ and $A\left(g, v_{i}\right)$ are defined in (2-14) and (2-15) respectively. Multiply (2-25) by $v_{i}^{2}\left(y_{i}\right)$ and let $i \rightarrow \infty$. Using Corollary 2.11, Lemma 2.4, and Corollary 2.13, one has

(2-26)

$$
\lim _{r \rightarrow 0^{+}} \int_{\Gamma_{2}\left(B_{r}^{+}\right)} B(r, x, h, \nabla h)=\lim _{r \rightarrow 0^{+}} \limsup _{i \rightarrow \infty} v_{i}^{2}\left(y_{i}\right) \int_{\Gamma_{2}\left(B_{r}^{+}\right)} B\left(r, x, v_{i}, \nabla v_{i}\right) \geq 0 .
$$

On the other hand, a direct calculation yields

$$
\lim _{r \rightarrow 0^{+}} \int_{\Gamma_{2}\left(B_{r}^{+}\right)} B(r, x, h, \nabla h)=-c A
$$

for some $c>0$. The conclusion follows from (2-26) and (2-27).

Now we can prove that an isolated blow-up point is in fact an isolated simple blow-up point.

Proposition 2.15. Let $v_{i}$ satisfy $\left(\mathrm{P}_{i}\right)$ and $y_{i} \rightarrow \bar{y}$ be an isolated blow-up point. Then $\bar{y}$ must be an isolated simple blow-up point.

Proof. The proof is much the same as that of [Felli and Ould Ahmedou 2003, Prop. 2.11]. For the reader's convenience, we include it here. From Lemma 2.4, it follows that

$$
\bar{w}_{i}{ }^{\prime}(r)<0 \quad \text { for every } C_{2} v_{i}^{1-q_{i}}\left(y_{i}\right) \leq r \leq r_{i} \text {. }
$$


Suppose that the blow-up is not simple; then there exist sequences $\tilde{r}_{i} \rightarrow 0^{+}$and $\tilde{c}_{i} \rightarrow+\infty$ such that $\tilde{c}_{i} v_{i}^{1-q_{i}}\left(y_{i}\right) \leq \tilde{r}_{i}$ and, after passing to a subsequence,

$$
\bar{w}_{i}^{\prime}\left(\tilde{r}_{i}\right) \geq 0 .
$$

From (2-28) and (2-29) it is clear that $\tilde{r}_{i} \geq r_{i}$ and $\bar{w}_{i}$ has at least one critical point in the interval $\left[r_{i}, \tilde{r}_{i}\right]$. Let $\mu_{i}$ be the smallest critical point of $\bar{w}_{i}$ in this interval. We have

$$
\tilde{r}_{i} \geq \mu_{i} \geq r_{i} \quad \text { and } \quad \lim _{i \rightarrow \infty} \mu_{i}=0 .
$$

Let $g_{i}=\left(g_{i}\right)_{\alpha \beta} d x^{\alpha} d x^{\beta}=g_{\alpha \beta}\left(\mu_{i} x\right) d x^{\alpha} d x^{\beta}$ be the scaled metric and set

$$
\xi_{i}(x)=\mu_{i}^{1 /\left(q_{i}-1\right)} v_{i}\left(y_{i}+\mu_{i} x\right) .
$$

Then $\xi_{i}$ satisfies

$$
\begin{cases}-\Delta_{g_{i}} \xi_{i}+\frac{1}{8} R_{g_{i}} \xi_{i}=0 & \text { in } B_{1 / \mu_{i}}^{-T_{i}}, \\ \frac{\partial \xi_{i}}{\partial v}=\widetilde{H}_{i}(x) \xi^{q_{i}} \xi_{i}^{q_{i}} & \text { on } \partial B_{1 / \mu_{i}}^{-T_{i}} \cap\left\{x^{3}=-T_{i}\right\} ;\end{cases}
$$

$\lim _{i \rightarrow \infty} \xi_{i}(0)=\infty$ and 0 is a local maximum point of $\xi_{i}$; also $r^{1 /\left(q_{i}-1\right)} \bar{\xi}_{i}(r)$ has negative derivative in $c \xi_{i}(0)^{1-q_{i}}<r<1$ and

$$
\left.\frac{d}{d r}\left(r^{1 /\left(q_{i}-1\right)} \bar{\xi}_{i}(r)\right)\right|_{r=1}=0
$$

where $T_{i}=\mu_{i}^{-1} y_{i}^{3}, \tilde{a}_{i}(x)=\mu_{i} a_{i}\left(y_{i}+\mu_{i} x\right)$, and $\tilde{H}_{i}(x)=H_{i}\left(y_{i}+\mu_{i} x\right)$. Arguing as in the proof of Lemma 2.4, we can easily prove that $T_{i} \rightarrow 0$. Since 0 is an isolated simple blow-up point, by Proposition 2.5 and Lemma 2.3, we have, for some $\beta>0$,

$$
\xi_{i}(0) \xi_{i} \underset{i \rightarrow+\infty}{\longrightarrow} h=\beta|x|^{-1}+E \quad \text { in } C_{\mathrm{loc}}^{2}\left(\mathbb{R}_{+}^{3} \backslash\{0\}\right),
$$

with $E$ satisfying

$$
\begin{cases}-\Delta E=0, & \text { in } \mathbb{R}_{+}^{3}, \\ \frac{\partial E}{\partial v}=0, & \text { on } \partial \mathbb{R}_{+}^{3}\end{cases}
$$

By the maximum principle we have $E \geq 0$. Reflecting $E$ to be defined on all $\mathbb{R}^{3}$ and thus using Liouville's Theorem, we deduce that $E$ is a constant. Using (2-30) and (2-31), we deduce that $E \equiv b$. Therefore $h(x)=b\left(G_{a}(x, \bar{y})+1\right)$, contradicting Proposition 2.14. 


\section{Ruling out bubble accumulations}

Now we can proceed as in [Felli and Ould Ahmedou 2003] to obtain the following results, which rule out possible accumulations of bubbles, thus implying that only isolated blow-up points may occur for blowing-up sequences of solutions.

Proposition 3.1. Let $(M, g)$ be a smooth compact three-dimensional Riemannian manifold with umbilic boundary. For any $R \geq 1$ and $0<\varepsilon<1$, there exist positive constants $\delta_{0}, c_{0}$, and $c_{1}$ depending only on $M, g,\|H\|_{C^{2}(\partial M)}$, $\inf _{y \in \partial M} H(y), R$, and $\varepsilon$, such that for all $u$ in

$$
\bigcup_{3-\delta_{0} \leq q \leq 3} M_{H, q}
$$

with $\max _{M} u \geq c_{0}$ there exists $\mathscr{Y}=\left\{p_{1}, \ldots, p_{N}\right\} \subset \partial M$ with $N \geq 1$ satisfying the following conditions:

(i) each $p_{i}$ is a local maximum point of $u$ in $M$ and

$$
\bar{B}_{\bar{r}_{i}}\left(p_{i}\right) \cap \bar{B}_{\bar{r}_{j}}\left(p_{j}\right)=\varnothing, \quad \text { for } i \neq j,
$$

where $\bar{r}_{i}=R u^{1-q}\left(p_{i}\right)$ and $\bar{B}_{\bar{r}_{i}}\left(p_{i}\right)$ denotes the geodesic ball in $(M, g)$ of radius $\bar{r}_{i}$ and centered at $p_{i}$;

$$
\left\|u^{-1}\left(p_{i}\right) u\left(\exp _{p_{i}}\left(y u^{1-q}\left(p_{i}\right)\right)\right)-\sqrt{\frac{1}{\left(1+h x^{3}\right)^{2}+h^{2}\left|x^{\prime}\right|^{2}}}\right\|_{C^{2}\left(B_{2 R}^{M}(0)\right)}<\varepsilon,
$$

where

$$
\begin{aligned}
B_{2 R}^{M}(0) & =\left\{y \in T_{p_{i}} M:|y| \leq 2 R, u^{1-q}\left(p_{i}\right) y \in \exp _{p_{i}}^{-1}\left(B_{\delta}\left(p_{i}\right)\right)\right\}, \\
y=\left(y^{\prime}, y^{n}\right) & \in \mathbb{R}^{n}, \text { and } h>0 ;
\end{aligned}
$$

(iii) $d^{1 /(q-1)}\left(p_{j}, p_{i}\right) u\left(p_{j}\right) \geq c_{0}$, for $j>i$, while $d(p, \mathscr{Y})^{1 /(q-1)} u(p) \leq c_{1}$, for all $p \in M$, where $d(\cdot, \cdot)$ denotes the distance function in metric $g$.

Proposition 3.2. Let $(M, g)$ be a smooth compact three-dimensional Riemannian manifold with umbilic boundary. For suitably large $R$ and small $\varepsilon>0$, there exist $\delta_{1}$ and $d$ depending only on $M, g,\|a\|_{C^{2}(\partial M)},\|H\|_{C^{2}(\partial M)}, \inf _{y \in \partial M} H(y), R$, and $\varepsilon$, such that for all $u$ in $\bigcup_{3-\delta_{1} \leq q \leq 3} M_{a, H, q}$ with $\max _{M} u \geq c_{0}$, we have

$$
\min \left\{d\left(p_{i}, p_{j}\right): i \neq j, 1 \leq i, j \leq N\right\} \geq d
$$

where $c_{0}, p_{1}, \ldots, p_{N}$ are given by Proposition 3.1.

The previous two propositions imply that any blow-up point is in fact an isolated blow-up point. Thanks to Proposition 2.15, any blow-up point is in fact an isolated simple blow-up point.

Proof of Theorem 1.1. Arguing by contradiction, suppose that there exist sequences $q_{i} \rightarrow q \in(1,3], u_{i} \in M_{H_{i}, q_{i}}$ such that $\left\|u_{i}\right\|_{H^{1}(M)} \rightarrow+\infty$ as $i \rightarrow \infty$, which, in view of standard elliptic estimates, implies that $\max _{M} u_{i} \rightarrow+\infty$. 
From [Hu 1994] (see also [Li and Zhang 2003]), we know that $q=3$. By Proposition 3.2, for some small $\varepsilon>0$, large $R>0$, and some $N \geq 1$ there exist $y_{i}^{(1)}, \ldots, y_{i}^{(N)} \in \partial M$ such that conditions (i)-(iii) Proposition 3.1 hold. The points $\left\{y_{i}^{(1)}\right\}_{i}, \ldots,\left\{y_{i}^{(N)}\right\}_{i}$ are isolated blow-up points and hence, by Proposition 2.15, isolated simple blow-up points. From (2-2) and Proposition 2.5, the sequence $\left\{\left\|u_{i}\right\|_{H^{1}(M)}\right\}_{i}$ is bounded, which gives a contradiction.

\section{Compactness of the solutions}

Before proving Theorem 1.2, we state the following result about the compactness of solutions of $\left(\mathrm{P}_{H, q}\right)$ when $q$ stays strictly below the critical exponent. The proof is basically the same as that of [Felli and Ould Ahmedou 2003, Theorem 3.1].

Theorem 4.1. Let $(M, g)$ be a smooth compact three-dimensional Riemannian manifold with umbilic boundary. Then for any $\delta_{1}>0$ there exists a constant $C>0$ depending only on $M, g, \delta_{1},\|H\|_{C^{2}(\partial M)}$, and the positive lower bound of $H$ on $\partial M$ such that for all $u \in \bigcup_{1+\delta_{1} \leq q \leq 3-\delta_{1}} M_{H, q}$ we have

$$
\|u\|_{C^{2}(M)} \leq C \quad \text { and } \quad \frac{1}{C} \leq u(x) \leq C \quad \text { for all } x \in M .
$$

Proof of Theorem 1.2. Due to elliptic estimates and Lemma 2.3, we have to prove just the $L^{\infty}$ bound, i.e., $u \leq C$. Suppose the contrary; then there exists a sequence $q_{i} \rightarrow q \in(1,3]$ with

$$
u_{i} \in M_{H, q_{i}} \text { and } \max _{M} u_{i} \rightarrow+\infty,
$$

where $\bar{c}$ is some positive constant independent of $i$. From Theorem 4.1, q must be 3 . It follows from Propositions 2.15 and 3.2 that, after passing to a subsequence, $\left\{u_{i}\right\}_{i}$ has $N$ (with $1 \leq N<\infty$ ) isolated simple blow-up points denoted by $y^{(1)}, \ldots, y^{(N)}$. Let $y_{i}^{(\ell)}$ denote the local maximum points as in Definition 2.1. It follows from Proposition 2.5 that $u_{i}\left(y_{i}^{(1)}\right) u_{i} \underset{i \rightarrow+\infty}{\longrightarrow} h(y)=\sum_{j=1}^{N} b_{j} G\left(y, y^{(j)}\right)+E(y) \quad$ in $C_{\mathrm{loc}}^{2}\left(M \backslash\left\{y^{(1)}, \ldots, y^{(N)}\right\}\right)$, where $b_{j}>0$ and $E \in C^{2}(M)$ satisfies

$$
\begin{cases}-L_{g} E=0 & \text { in } M \\ \frac{\partial E}{\partial v}=0 & \text { on } \partial M\end{cases}
$$

Since the manifold is of positive type, $E \equiv 0$. Therefore,

$$
u_{i}\left(y_{i}^{(1)}\right) u_{i} \underset{i \rightarrow+\infty}{\longrightarrow} h(y)=\sum_{j=1}^{N} b_{j} G_{a}\left(y, y^{(j)}\right) \quad \text { in } C_{\mathrm{loc}}^{2}\left(M \backslash\left\{y^{(1)}, \ldots, y^{(N)}\right\}\right) .
$$


Let $x=\left(x^{1}, x^{2}, x^{3}\right)$ be a geodesic normal coordinate system centered at $y_{i}^{(1)}$. From Lemma A.5, the Positive Mass Theorem, and the assumption that the manifold is not conformally equivalent to the standard ball, we derive that there exists a positive constant $A$ such that

$$
h(x)=h\left(\exp _{y_{i}^{(1)}}(x)\right)=c|x|^{-1}+A_{i}+O\left(|x|^{-\alpha}\right) \text { for }|x| \text { close to } 0
$$

and $A_{i} \geq A>0$. This contradicts the result of Proposition 2.14. The compactness part of Theorem 1.2 is proved. Once we have compactness, we can proceed as in [Felli and Ould Ahmedou 2003, Section 4] to prove that the total degree of the solutions is -1 .

\section{Appendix}

Here we recall some needed results and describe the singular behaviour of positive solutions to certain boundary value elliptic equations in punctured half-balls.

For $n \geq 3$, let $B_{r}^{+}:\left\{x=\left(x^{\prime}, x^{n}\right) \in \mathbb{R}^{n}=\mathbb{R}^{n-1} \times \mathbb{R}:|x|<r\right.$ and $\left.x^{n}>0\right\}$ and set $\Gamma_{1}\left(B_{r}^{+}\right):=\partial B_{r}^{+} \cap \partial \mathbb{R}_{+}^{n}, \Gamma_{2}\left(B_{r}^{+}\right):=\partial B_{r}^{+} \cap \mathbb{R}_{+}^{n}$. Consider a smooth Riemannian metric $g=g_{i j} d x^{i} d x^{j}$ in $B_{1}^{+}$, and $a \in C^{1}\left(\Gamma_{1}\left(B_{1}^{+}\right)\right)$.

We first recall a maximum principle; for the proof see [Han and Li 1999].

Theorem A.1. Let $\Omega$ be a bounded domain in $\mathbb{R}^{n}$ and let $\partial \Omega=\Gamma \cup \Sigma, V \in L^{\infty}(\Omega)$, and $h \in L^{\infty}(\Sigma)$ be such that there exists $\psi \in C^{2}(\Omega) \cap C^{1}(\bar{\Omega})$ positive in $\bar{\Omega}$ and satisfying

$$
\begin{cases}\Delta_{g} \psi+V \psi \leq 0 & \text { in } \Omega \\ \frac{\partial \psi}{\partial v} \geq h \psi & \text { on } \Sigma .\end{cases}
$$

If $v \in C^{2}(\Omega) \cap C^{1}(\bar{\Omega})$ satisfies

$$
\begin{cases}\Delta_{g} v+V v \leq 0 & \text { in } \Omega, \\ \frac{\partial v}{\partial v} \geq h v & \text { on } \Sigma, \\ v \geq 0, & \text { on } \Gamma,\end{cases}
$$

then $v \geq 0$ in $\bar{\Omega}$.

Next we state a maximum principle that holds for the operator $T$ defined by

$$
T u=v \text { if and only if } \begin{cases}L_{g} u=0 & \text { in } \stackrel{\circ}{\partial u}, \\ \frac{\partial v}{\partial v}=v & \text { on } \partial M .\end{cases}
$$

Proposition A.2 [Escobar 1996]. Let $(M, g)$ be a Riemannian manifold with boundary of positive type. Then, for any $u \in C^{2}(\stackrel{\circ}{M}) \cap C^{1}(M)$ satisfying $L_{g} u \geq 0$ in $\stackrel{\circ}{M}$ and $\partial u / \partial v \leq 0$ on $\partial M$, we have $u \leq 0$ in $M$. 
Proof. Let $u^{+}(x)=\max \{0, u(x)\}$. Then

$$
0 \leq \int_{M}\left(L_{g} u\right) u^{+} d V-\int_{\partial M} \frac{\partial u}{\partial v} u^{+} d \sigma=-\int_{M}\left|\nabla_{g} u^{+}\right|^{2} d V-\frac{1}{8} \int_{M} R_{g}^{2}\left|u^{+}\right|^{2} d V .
$$

Since $M$ is of positive type $\int\left|\nabla_{g} u\right|^{2}+\frac{1}{8} \int R_{g} u^{2}$ is an equivalent norm, hence $u^{+} \equiv 0$.

We now recall a Louville-type theorem, from [Li and Zhu 1995]. See also [Escobar 1990; Chipot et al. 1996].

Theorem A.3. If $v$ is a solution of

$$
\begin{cases}-\Delta v=0 & \text { in } \mathbb{R}_{+}^{n}, \\ \frac{\partial v}{\partial x^{n}}=c v^{n /(n-2)} & \text { on } \partial \mathbb{R}_{+}^{n},\end{cases}
$$

and $c$ is a negative constant, then either $v \equiv 0$ or $v$ is of the form

$$
v\left(x^{\prime}, x^{n}\right)=\left(\frac{\varepsilon}{\left(x_{0}^{n}+x^{n}\right)^{2}+\left|x^{\prime}-x_{0}^{\prime}\right|^{2}}\right)^{\frac{n-2}{2}} \quad \text { for } x^{\prime} \in \mathbb{R}^{n-1}, x^{n} \in \mathbb{R},
$$

where $x_{0}^{n}=-(n-2) \varepsilon / c$, for some $\varepsilon>0$, and $x_{0}^{\prime} \in \mathbb{R}^{n-1}$.

Lemma A.4. Suppose that $u \in C^{2}\left(B_{1}^{+} \backslash\{0\}\right)$ is a solution of

$$
\begin{cases}-L_{g} u=0, & \text { on } B_{1}^{+}, \\ \frac{\partial u}{\partial v}=0, & \text { on } \Gamma_{1}\left(B_{1}^{+} \backslash\{0\}\right),\end{cases}
$$

and $u(x)=o\left(|x|^{2-n}\right)$ as $|x| \rightarrow 0$. Then $u \in C^{2, \alpha}\left(B_{1 / 2}^{+}\right)$for any $0<\alpha<1$.

Proof. We reflect across $\Gamma_{1}\left(B_{1}^{+}\right)$to extend $u$ as a solution of $-L_{g} u=0$ on $B_{1} \backslash\{0\}$, then use [Gilbarg and Serrin 1955/56] to conclude that 0 is a removable singularity. The result follows from standard elliptic regularity.

Lemma A.5. There exists some constant $\delta_{0}>0$ depending only on $n,\left\|g_{i j}\right\|_{C^{2}\left(B_{1}^{+}\right)}$ and $\|H\|_{L^{\infty}\left(B_{1}^{+}\right)}$such that for all $0<\delta<\delta_{0}$ there exists some function $G$ satisfying

$$
\begin{cases}-L_{g} G=0, & \text { in } B_{\delta}^{+}, \\ \frac{\partial G}{\partial v}=0, & \text { on } \Gamma_{1}\left(B_{\delta}^{+}\right) \backslash\{0\}, \\ \lim _{|x| \rightarrow 0}|x|^{-1} G(x)=1 & \end{cases}
$$

such that, for some constant $A$ and some $\alpha \in(0,1)$,

$$
G(x)=|x|^{-1}+A+O\left(|x|^{\alpha}\right) \quad \text { for all } x \in B_{\delta}^{+} .
$$

Proof. Reflecting across $\Gamma_{1}\left(B_{\delta}^{+}\right)$, the lemma is reduced to [Li and Zhu 1997, Proposition B.1]. 
Reflecting again across $\Gamma_{1}\left(B_{1}^{+}\right)$, we derive from [Li and Zhu 1999, Lemma 9.3] the following result:

Lemma A.6. Assume that $u \in C^{2}\left(B_{1}^{+} \backslash\{0\}\right)$ satisfies

$$
\begin{cases}-L_{g} u=0 & \text { in } B_{1}^{+} \\ \frac{\partial u}{\partial v}=0 & \text { on } \Gamma_{1}\left(B_{1}^{+}\right) \backslash\{0\} .\end{cases}
$$

Then

$$
\alpha=\limsup _{r \rightarrow 0^{+}} \max _{x \in \Gamma_{2}\left(B_{r}^{+}\right)} u(x)|x|^{n-2}<+\infty .
$$

Proposition A.7. Suppose that $u \in C^{2}\left(B_{1}^{+} \backslash\{0\}\right)$ satisfies

$$
\begin{cases}-L_{g} u=0 & \text { in } B_{1}^{+} \\ \frac{\partial u}{\partial v}=0 & \text { on } \Gamma_{1}\left(B_{1}^{+}\right) \backslash\{0\} .\end{cases}
$$

Then there exists some constant $b \geq 0$ such that

$$
u(x)=b G(x)+E(x) \quad \text { in } B_{1 / 2}^{+} \backslash\{0\},
$$

where $G$ is defined in Lemma A.5, and $E \in C^{2}\left(B_{1}^{+}\right)$satisfies

$$
\left\{\begin{array}{l}
-L_{g} E=0 \quad \text { in } B_{1 / 2}^{+} \\
\frac{\partial E}{\partial v}=0 \quad \text { on } \Gamma_{1}\left(B_{1 / 2}^{+}\right) .
\end{array}\right.
$$

Proof. Set

$$
b=b(u)=\sup \left\{\lambda \geq 0: \lambda G \leq u \text { in } \bar{B}_{\delta_{0}}^{+} \backslash\{0\}\right\} .
$$

By the previous lemma we know that $0 \leq b \leq \alpha<+\infty$. Two cases may occur.

Case 1: $b=0$. We claim that for all $\varepsilon>0$ there exists $r_{\varepsilon} \in\left(0, \delta_{0}\right)$ such that

$$
\min _{x \in \Gamma_{2}\left(B_{r}^{+}\right)}\{u(x)-\varepsilon G(x)\} \leq 0 \text { for any } 0<r<r_{\varepsilon} .
$$

For suppose otherwise. Then there exist $\varepsilon_{0}>0$ and a sequence $r_{j} \rightarrow 0^{+}$such that

$$
\min _{|x|=r_{j}}\left\{u(x)-\varepsilon_{0} G(x)\right\}>0 \text { and } u(x)-\varepsilon_{0} G(x)>0 \text { on } \Gamma_{2}\left(B_{\delta_{0}}^{+}\right) .
$$

We prove that $\varepsilon_{0} \leq b$, contradicting the assumptions. To do this note that

$$
\begin{cases}-\Delta_{g}\left(u-\varepsilon_{0} G\right)+\frac{1}{8} R_{g}\left(u-\varepsilon_{0} G\right)=0 & \text { in } B_{\delta_{0}}^{+}, \\ \frac{\partial}{\partial v}\left(u-\varepsilon_{0} G\right)=0 & \text { on } \Gamma_{1}\left(B_{\delta_{0}}^{+}\right) \backslash\{0\},\end{cases}
$$

and apply Theorem A.1 with

$$
v=u-\varepsilon_{0} G, \quad \Sigma=\Gamma_{1}\left(B_{\delta_{0}}^{+}\right) \backslash \Gamma_{1}\left(B_{r_{j}}^{+}\right), \quad \Gamma=\Gamma_{2}\left(B_{r_{j}}^{+}\right) \cup \Gamma_{2}\left(B_{\delta_{0}}^{+}\right) ;
$$


this yields $u-\varepsilon_{0} G \geq 0$ in the annulus $B_{\delta_{0}}^{+} \backslash B_{r_{j}}^{+}$for any $j$, and consequently in $B_{\delta_{0}}^{+} \backslash\{0\}$. Therefore $\varepsilon_{0} \leq b$ and we have our contradiction.

Hence, for any $\varepsilon>0$ and $0<r<r_{\varepsilon}$ there exists $x_{\varepsilon} \in \Gamma_{2}\left(B_{r}^{+}\right)$such that

$$
u\left(x_{\varepsilon}\right) \leq \varepsilon G\left(x_{\varepsilon}\right) .
$$

By the Harnack inequality of Lemma 2.3 we have that

$$
\max _{|x|=r} u(x) \leq c u\left(x_{\varepsilon}\right) \leq c \varepsilon G\left(x_{\varepsilon}\right) .
$$

Since $G(x) \sim|x|^{2-n}$ for $|x|$ small, we conclude that

$$
u(x)=o\left(|x|^{2-n}\right) \quad \text { for }|x| \sim 0 .
$$

Therefore from Lemma A.4 we obtain that $u$ is regular. Setting $E(x)=u(x)$, the conclusion in this case follows.

Case 2. $b>0$.

We consider $v(x)=u(x)-b G(x)$ in $\bar{B}_{\delta_{0}}^{+} \backslash\{0\}$. By definition of $b$, it is clear that $v \geq 0$ in $\bar{B}_{\delta_{0}}^{+} \backslash\{0\}$. Moreover $v$ satisfies

$$
\begin{cases}-\Delta_{g} v+\frac{1}{8} R_{g} v=0, & \text { in } B_{\delta_{0}}^{+}, \\ \frac{\partial v}{\partial v}=0, & \text { on } \Gamma_{1}\left(B_{\delta_{0}}^{+}\right) \backslash\{0\},\end{cases}
$$

so that from the maximum principle we know that either $v \equiv 0$ or $v>0$ in $B_{\delta_{0}}^{+} \backslash\{0\}$. If $v \equiv 0$, take $E \equiv 0$ and we are done. Otherwise $v>0$ and satisfies the same equation as $u$. Set

$$
\tilde{b}=b(v)=\sup \left\{\lambda \geq 0: \lambda G \leq v \text { in } \bar{B}_{\delta_{0}}^{+} \backslash\{0\}\right\} .
$$

If $\lambda \geq 0$ and $\lambda G \leq v$ in $B_{\delta_{0}}^{+} \backslash\{0\}$, then $\lambda G \leq u-b G$ with $b>0$, i.e. $(\lambda+b) G \leq u$. By the definition of $b$, this implies that $\lambda+b \leq b$, so $\lambda=0$. Therefore $\tilde{b}=0$. Arguing as in Case 1, we can prove that $v(x)=o\left(|x|^{2-n}\right)$ for $|x| \sim 0$. Lemma A.4 ensures that $v$ is regular, so that choosing $E(x)=v(x)$ we are done.

The proof of Proposition A.7 is thereby complete.

Corollary A.8. Let $u$ be a solution of (A-3) which is singular at 0 . Then

$$
\lim _{r \rightarrow 0^{+}} \int_{\Gamma_{2}\left(B_{r}^{+}\right)} \frac{\partial u}{\partial v} d \sigma=b \lim _{r \rightarrow 0^{+}} \int_{\Gamma_{2}\left(B_{r}^{+}\right)} \frac{\partial G}{\partial v} d \sigma=-\frac{n-2}{2} b\left|\mathbb{S}^{n-1}\right|,
$$

where $\mathbb{S}^{n-1}$ denotes the standard $n$-dimensional sphere and $b>0$ is given by Proposition A.7.

Proof. From the previous proposition, we know that

$$
u(x)=b G(x)+E(x) \quad \text { in } B_{1 / 2}^{+}(0) \backslash\{0\}, \quad \text { with } b \geq 0 .
$$


Since $u$ is singular at $0, b$ must be strictly positive. From (A-4), we have

$$
0=-\int_{B_{r}^{+}} \Delta_{g} E d V-\int_{\Gamma_{2}\left(B_{r}^{+}\right)} \frac{\partial E}{\partial v} d \sigma+\frac{1}{8} \int_{B_{r}^{+}} R_{g} E .
$$

Hence, since $E$ is regular, we obtain

$$
\int_{\Gamma_{2}\left(B_{r}^{+}\right)} \frac{\partial E}{\partial v} d \sigma=\frac{1}{8} \int_{B_{r}^{+}} R_{g}(x) E(x) d \sigma \underset{r \rightarrow 0^{+}}{\longrightarrow} 0
$$

and so

$$
\lim _{r \rightarrow 0^{+}} \int_{\Gamma_{2}\left(B_{r}^{+}\right)} \frac{\partial u}{\partial v} d \sigma=\lim _{r \rightarrow 0^{+}} b \int_{\Gamma_{2}\left(B_{r}^{+}\right)} \frac{\partial G}{\partial v} d \sigma .
$$

From Lemma A.5 we know that $G$ is of the form

$$
G(x)=|x|^{-1}+\mathscr{R}(x),
$$

where $\mathscr{R}$ is regular. Since

$$
\int_{\Gamma_{2}\left(B_{r}^{+}\right)} \frac{\partial}{\partial v}|x|^{-1} d \sigma=-\frac{1}{2}\left|\mathbb{S}^{n-1}\right|
$$

and

$$
\int_{\Gamma_{2}\left(B_{r}^{+}\right)} \frac{\partial \mathscr{R}}{\partial v} d \sigma \underset{r \rightarrow+0^{+}}{\longrightarrow} 0
$$

we conclude that

$$
\lim _{r \rightarrow 0^{+}} \int_{\Gamma_{2}\left(B_{r}^{+}\right)} \frac{\partial u}{\partial v} d \sigma=-\frac{1}{2} b\left|\mathbb{S}^{n-1}\right|
$$

and the corollary follows.

\section{Acknowledgements}

We thank the referee for a careful reading, and for useful comments and suggestions. We are grateful to Prof. A. Ambrosetti and Prof. Alice Chang for their interest in this work and for their constant support. Ahmedou thanks Prof. Reiner Schätzle for useful discussions when preparing a final version of this paper.

\section{References}

[Bahri 1989] A. Bahri, Critical points at infinity in some variational problems, Pitman Research Notes in Mathematics Series 182, Longman, Harlow, 1989. MR 91h:58022 Zbl 0676.58021

[Bahri and Brezis 1996] A. Bahri and H. Brezis, "Non-linear elliptic equations on Riemannian manifolds with the Sobolev critical exponent", pp. 1-100 in Topics in geometry, edited by S. Gindikin, Progr. Nonlinear Differential Equations Appl. 20, Birkhäuser, Boston, MA, 1996. MR 97c:53056

[Bahri and Coron 1988] A. Bahri and J.-M. Coron, "On a nonlinear elliptic equation involving the critical Sobolev exponent: the effect of the topology of the domain", Comm. Pure Appl. Math. 41:3 (1988), 253-294. MR 89c:35053 Zbl 0649.35033

[Bartz et al. 1994] J. Bartz, M. Struwe, and R. Ye, "A new approach to the Ricci flow on $S^{2}$ ", Ann. Scuola Norm. Sup. Pisa Cl. Sci. (4) 21:3 (1994), 475-482. MR 95i:53039 Zbl 0818.53050 
[Brendle 2002a] S. Brendle, "Curvature flows on surfaces with boundary", Math. Ann. 324:3 (2002), 491-519. MR 2003j:53103 Zbl 1024.53045

[Brendle 2002b] S. Brendle, "A family of curvature flows on surfaces with boundary", Math. Z. 241:4 (2002), 829-869. MR 2003j:53104 Zbl 1036.53044

[Chang et al. 1998] S.-Y. A. Chang, X. Xu, and P. C. Yang, "A perturbation result for prescribing mean curvature", Math. Ann. 310:3 (1998), 473-496. MR 2000a:58091 Zbl 0893.35033

[Chen 2001] X. X. Chen, "Calabi flow in Riemann surfaces revisited: a new point of view", Internat. Math. Res. Notices 2001:6 (2001), 275-297. MR 2002c:53111

[Chipot et al. 1996] M. Chipot, I. Shafrir, and M. Fila, "On the solutions to some elliptic equations with nonlinear Neumann boundary conditions", Adv. Differential Equations 1:1 (1996), 91-110. MR 96h:35059 Zbl 0839.35042

[Chow 1991] B. Chow, "The Ricci flow on the 2-sphere", J. Differential Geom. 33:2 (1991), 325334. MR 92d:53036 Zbl 0734.53033

[Djadli et al. 2004] Z. Djadli, A. Malchiodi, and M. Ould Ahmedou, "The prescribed boundary mean curvature problem on $\mathbb{B}^{4,}$, J. Diff. Eq. 206 (2004), 373-398.

[Escobar 1990] J. F. Escobar, "Uniqueness theorems on conformal deformation of metrics, Sobolev inequalities, and an eigenvalue estimate", Communications Pure Appl. Math. 43:7 (1990), 857-883. MR 92f:58038 Zbl 0713.53024

[Escobar 1992a] J. F. Escobar, "Conformal deformation of a Riemannian metric to a scalar flat metric with constant mean curvature on the boundary", Ann. of Math. (2) 136:1 (1992), 1-50. MR 93e:53046 Zbl 0766.53033

[Escobar 1992b] J. F. Escobar, "The Yamabe problem on manifolds with boundary", J. Differential Geom. 35:1 (1992), 21-84. MR 93b:53030 Zbl 0771.53017

[Escobar 1996] J. F. Escobar, "Conformal metrics with prescribed mean curvature on the boundary", Calc. Var. Partial Differential Equations 4:6 (1996), 559-592. MR 97h:53040 Zbl 0867.53034

[Escobar and Garcia 2004] J. F. Escobar and G. Garcia, "Conformal metrics on the ball with zero scalar curvature and prescribed mean curvature on the boundary", J. Funct. Anal. 211:1 (2004), 71-152. MR 2054619

[Felli and Ould Ahmedou 2003] V. Felli and M. Ould Ahmedou, "Compactness results in conformal deformations of Riemannian metrics on manifolds with boundaries", Math. Z. 244:1 (2003), 175210. MR 2004d:53034 Zbl 01944238

[Gilbarg and Serrin 1955/56] D. Gilbarg and J. Serrin, "On isolated singularities of solutions of second order elliptic differential equations", J. Analyse Math. 4 (1955/56), 309-340. MR 18,399a Zbl 0071.09701

[Hamilton 1988] R. S. Hamilton, "The Ricci flow on surfaces", pp. 237-262 in Mathematics and general relativity (Santa Cruz, CA, 1986), edited by J. A. Isenberg, Contemp. Math. 71, Amer. Math. Soc., Providence, RI, 1988. MR 89i:53029 Zbl 0663.53031

[Han and Li 1999] Z.-C. Han and Y. Li, "The Yamabe problem on manifolds with boundary: existence and compactness results", Duke Mathematical J. 99:3 (1999), 489-542. MR 2000j:53045 Zbl 0945.53023

[Hu 1994] B. Hu, "Nonexistence of a positive solution of the Laplace equation with a nonlinear boundary condition", Differential Integral Equations 7:2 (1994), 301-313. MR 95a:35047 Zbl 0820.35062

[Li 1995] Y. Y. Li, "Prescribing scalar curvature on $S^{n}$ and related problems. I", J. Differential Equations 120:2 (1995), 319-410. MR 98b:53031 Zbl 0827.53039

[Li 1996] Y. Li, "Prescribing scalar curvature on $S^{n}$ and related problems, II: Existence and compactness", Comm. Pure Appl. Math. 49:6 (1996), 541-597. MR 98f:53036 Zbl 0849.53031 
[Li and Zhang 2003] Y. Li and L. Zhang, "Liouville-type theorems and Harnack-type inequalities for semilinear elliptic equations", J. Anal. Math. 90 (2003), 27-87. MR 2004i:35118

[Li and Zhu 1995] Y. Li and M. Zhu, "Uniqueness theorems through the method of moving spheres", Duke Math. J. 80:2 (1995), 383-417. MR 96k:35061 Zbl 0846.35050

[Li and Zhu 1997] Y. Li and M. Zhu, "Sharp Sobolev trace inequalities on Riemannian manifolds with boundaries", Communications Pure Appl. Math. 50:5 (1997), 449-487. MR 98c:46065 Zbl 0869.58054

[Li and Zhu 1999] Y. Li and M. Zhu, "Yamabe type equations on three-dimensional Riemannian manifolds", Commun. Contemp. Math. 1:1 (1999), 1-50. MR 2000m:53051 Zbl 0973.53029

[Ould Ahmedou 2003] M. Ould Ahmedou, "A Riemann mapping type theorem in higher dimensions, I: The conformally flat case with umbilic boundary", pp. 1-18 in Nonlinear equations: methods, models and applications (Bergamo, 2001), edited by D. Lupo et al., Progr. Nonlinear Differential Equations Appl. 54, Birkhäuser, Basel, 2003. MR 2023229 Zbl 1041.58010

[Schoen 1991] R. M. Schoen, "On the number of constant scalar curvature metrics in a conformal class", pp. 311-320 in Differential geometry, edited by H. B. Lawson and K. Tenenblat, Pitman Monogr. Surveys Pure Appl. Math. 52, Longman Sci. Tech., Harlow, 1991. MR 94e:53035 Zbl 0733.53021

[Struwe 2002] M. Struwe, "Curvature flows on surfaces", Ann. Sc. Norm. Super. Pisa Cl. Sci. (5) 1:2 (2002), 247-274. MR 2004f:53083

[Ye 1994] R. Ye, "Global existence and convergence of Yamabe flow", J. Differential Geom. 39:1 (1994), 35-50. MR 95d:53044 Zbl 0846.53027

Received July 26, 2001. Revised September 9, 2002.

\section{VERONICA FELLi}

UNIVERSITÀ DI MILANO BICOCCA

DipARTIMENTO Di MATEMATICA E APPLICAZIONI

Via COZZI 53, 20125 MiLANO

ITALY

felli@matapp.unimib.it

Mohameden Ould Ahmedou

EBERHARD-KARLS-UNIVERSITÄT TÜBINGEN

MATHEMATISCHES INSTITUT

AUf DER MORGENSTELLE , D-72076 TÜBINGEN

GERMANY

ahmedou@everest.mathematik.uni-tuebingen.de 